\title{
A performance enhancement of physical layer at Wi-MAX system with RLDPC code
}

\author{
Sandeep D. Bawage ${ }^{1}$, Manjula $S^{2}$, A. M. Bhavikatti ${ }^{3}$ \\ ${ }^{1,3}$ Department of ECE, LAEC, Bidar, Karnataka, India \\ ${ }^{2}$ Department of ECE, BKIT, Bhalki, Karnataka, India
}

\begin{tabular}{l} 
Article Info \\
Article history: \\
Received Jul 17, 2020 \\
Revised Oct 1, 2020 \\
Accepted Nov 11, 2020 \\
\hline Keywords: \\
Worldwide interoperability for \\
microwaves access \\
Multiple input multiple output \\
Physical layer \\
Orthogonal frequency division \\
multiplexing
\end{tabular}

\section{Article history:}

Received Jul 17, 2020

Revised Oct 1, 2020

Accepted Nov 11, 2020

\begin{abstract}
In present wireless communication network, the error correction codes plays the major role for efficient data transmission in noisy environments. To get minimum BER and PAPR has been the main aim towards the field in forward error control coding. Majority of the researchers has considered turbo codes at specific SNR over AWGN channel but have complexity issues with the iterative output decoder and causes degradation in the Wi-Max network system. In this paper, the author presents and evaluates WiMAX physical layer performance with using MIMO technologies, where a RobustLDPC technique of coding and decoding in OFDM based WiMAX system is considered. The decoding method of RLDPC has processed by Belief Propagation at the logarithmic domain in an iterative manner, the proposed methodology shows good decoding outcome for RLDPC codes at Rician and Rayleigh channel. Moreover, the applicability of our proposed model channel codes is defined under IEEE Wi-MAX standard and the results analysis is done under different code-rate and modulation schemes.
\end{abstract}

This is an open access article under the CC BY-SA license.

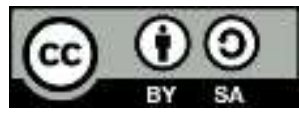

\section{Corresponding Author:}

Sandeep D Bawage

Department of Electronic and Communication Engineering

Lingaraj Appa Engineering College

Bidar, Karnataka, India

Email: sandeepbawage@gmail.com

\section{INTRODUCTION}

A WiMAX is highly foreseen technology that aims to deliver an efficient wireless broadband services to the consumers and business in form of MAN (metropolitan area network). IEEE 802.16e standard of WiMAX [1] is considered to be most emerging approach that support very large coverage area with the high speed data transmission. Due to its characteristics of data rate and high coverage area it fulfills the ideology of comprehensive network architecture. As per the advancement of wireless communication sector, stability, robustness, data transmission and reliability have become the continual optimization goal at wireless communication network.

Furthermore, in a network, MIMO technology is very effective for wireless communication that based upon the multiple antennas [2] and can be utilize channel very effectively without increasing bandwidth. This convert the effects of multipath to a favorable factors, which increase the wireless channel capacity, data transmission rate and utilization of spectrum [3]. Therefore, it causes improvement at performance and stability of communication system [4]. The OFDM process has significant development that aimed to impact at frequency selective fading due to its own large symbol cycle, so the combination of OFDM process and MIMO technologies in a present wireless communication network provide positive impact in the development area of wireless communication [5]. 
Considering OFDM-MIMO technology at WiMAX with standard 802.16e, there are several communication problems which co-exist. Where information is required to transfer from one node to another node via channel and it necessary that the received message should match with the original transmitted message. But unfortunately, problem arise during recovery of original signal due to occurrence of noise or errors during the process of transmission. The simple process to ensure data by correctly get such as iterative transmission, the difficulty with the process is it consume double as long in order to transmission information with the minimal errors. Therefore, the coding method of error control needs to increase the throughput with ensuring the correctly transmitted information.

The receiver side should get transmitter data that had sent through communication system, to achieve this redundancy approach must integrated to overcome errors which arises during process through channel. However, the errors can be instigated via the intrusion from different signals with noise, where difficulty is to obtain an acceptable error-rate without conceding the data-rate quality. So there is need to balance between 'data transmission-rate' and 'error-rate'.

In this paper, the author evaluates WiMAX 'physical layer performance' using MIMO technologies, where an efficient technique of coding and decoding in OFDM-WiMAX system is considered. The major aim of this research is to get maximum amount of data-rate (DR) with minimizing inconsequential error-rate (ER). PAPR (peak-to-average-power-ratio) and BER (bit-error-rate) result are presented using MIMOOFDM system, where coding and decoding schemes at different modulation process is considered. Here, the author have described the specification and properties of WiMAX, also the proposed RLDPC codes with belief propagation (BP) algorithm is presented. The results analysis section provide the performance analysis of MIMO-OFDM "physical layer" in the WiMAX technology by proposed RLDPC under different types of digital modulation techniques.

\section{LITERATURE SURVEY}

The Wi-MAX is a wireless broadband solution to access the mobile and static broadband networks via microwave access technology for WAN (wide area network). It provide high DR, scalability, quality of services and flexibility, where it make use of convolutional codes for the several type of modulation approach. Moreover, the Wi-MAX modulation technique make use of different sizes of the convolutional encoders and coding rates. In paper [6], authors had make efforts to analyze the effect at several modulation schemes, FFT size, cyclic prefix factors, and coding rates on the system performance. Also they studied the BER performance analysis of considered model at different channel scenarios.

While considering the wireless communication (WC) system, the turbo codes performance enhancement by error correction is mostly similar to Shannon limit [7], which is not possible for the convolutional codes at lower value of SNR [8]. However, several researchers have presented their area of interest in order to find optimal decoding algorithms for turbo codes implementation in real system scenarios, the turbo code delivers relatively optimized performance compared to convolution codes as described earlier. In [9], a basic BCJR algorithm is proposed for the maximum a posteriori ('MAP') decoding of forward error correction codes, decoding algorithm of BCJR MAP is computationally complex and sensitive to-wards the SNR mismatch, sometime provide inaccurate approximation of the noise variance. The turbo decoding based MAP algorithm propose the superlative performance with the iterative decoding process, but unable to decode until the entire bit sequence is achieved by receiver decoder [10]. The BCJR approach leads towards the large delay at decoding phase, more memory size and power consumption for iterations [11], therefore the iterative process of turbo decoding approach leads to maximize the complexity as compare to the conventional forward error correction (FEC) decoding approaches.

The iterative approach of decoding such as; MAP and SOV (soft output viterbi) algorithm requires the complex operations of decoding over some iteration cycles [12]. Whereas, the turbo code delivers an owing coding gain similar to Shannon limit at AWGN channel in order to achieve the maximum throughput for WiMAX system application [13]. In paper [14] provided the Mobile WiMAX system analysis by applying the turbo codes using SOV Algorithm, but SOV has the worse BER presentation and less computational complexity while the MAP algorithm delivers better BER performance and high computational complexity. Therefore, the turbo-code implementation in real time scenario, the reduction in decoder complexity is necessary with maintain BER performance at network [12].

In order to provide multicarrier data transmission, OFDM is the better choice is present generation network where OFDM divide high DR frames to several low DR frames. Though, the low DR frame having a sophisticated symbol period, which enables OFDM to decrease the multipath effect and impulse noise to a greater extent. However, the single carrier channel does not that capability to decrease the multipath effect, in [15] provide the study on OFDM system performance under some channel models such as; Multipath, AWGN and Rician. While considering OFDM based MIMO technology provides significant development in 
several WC systems and it got significant consideration from the researchers [16]. The combination of MIMO and OFDM delivers an optimized performance in terms of several parameters such as; BER, transmission rate, increased reliability and capacity, which causes high throughput rate at WLAN for 4G and 5G [17]. A compact version of inverted U-Shaped triple band MIMO antenna for WiMAX and WLAN application is given in [18].

The receiver and transmitter-based model [19] are simulated in accordance to parameters that recognized by standards, also the convolution code has been used to optimize the performance of system. Moreover, the Wi-Max system hardware implementation is done using FPGA (field programmable gate array) technology in [20]. In addition, they discussed about different algorithms of OFDM and the physical layer based WiMAX IEEE 802.16e standard at different groupings of digital modulation approaches and different channels of communication. A PAPR minimization of transmitted signal is also a major problem and this issue concern in an OFDM system, the usage interleaving is an attractive techniques that can be used in the reduction of PAPR for multicarrier signals. Without the spectrum distortion is possible but the communal drawback of interleaving methods is Side Information in transmission side to receiver side, and causes reduction in data rate. In [21], proposed a PAPR reduction approach using interleaving trade-off technique with an enhanced performances at PAPR reduction.

In a mobile Wi-MAX scenario where mobile nodes are travel at very high speed can cause a high challenge for management of QoS, however the multipath fading can causes more BER at receiver end is major cause for lower throughput at mobile nodes. Maximum packet size and BER define the error rate of packet, where the recovery of error for more number of tainted packets is not generally a good-looking option for several real-time network scenario with the jitter and delay constraints. Therefore, in [22] presented a mathematical model in order to get the bit error probability where the mobile nodes travels at various speeds. The multiple user's services with high DRs, Wi-MAX is most prominent technology that was presented by IEEE labelled for mobile WC and fixed wireless application. DSRC (dedicated-short range communication) proposed in [23], is the approach that trend in vehicular wireless network, where it applied on existing situations in Intelligent WC Transportation System and provides the 'high speed DRs'.

\section{RESEARCH METHOD}

The WiMAX is consider to standard technology which enables large area wireless broadband access, therefore its alternative to cable and DSL. The WiMAX technology is based upon the IEEE standard 802.16e-2005 which delivers multiple options from physical and MAC (medium access control) layer.

The MAC layer lies in between the physical layer and network layer, where physical layer be at top position and network layer at down position. MAC layer is used to provide access to wireless medium in order to transmit data frames. In according to some environmental conditions, the services and design specifications will change to accommodate various physical layer services and the protocol of MAC layer are designed to deliver point-to-multi-point services. Therefore, it decide the channel allocation algorithm as well as error control algorithm, this protocol labels the time and transmission way via user equipment or base station. In this study, the author majorly focusing on physical layer where the author want to minimize the BER by performing robust coding and decoding. Physical have the following specifications;

a) Randomizer: In process of data transmission in physical layer use randomizer on bit basis, each burst at the both side transmitter and receiver are equipped with randomized. The randomizing procedure done through scrambling and block is realized via the shift-registers.

b) Forward Error Correction: Distinctive feature of 'time-range' is generally performed by the optimized coding technique and used different streams of data which transmitted at each period through considering different 'code-words'. The consider robust LDPC-code has great operational flexibility because code can handle wide choice of rates, it also reduces BER and improve system accuracy.

c) Modulation Technique: The QAM provides modulation, used to map the incoming bits from interleaver to assemblage. Although coded bits from modulation are being mapped and then released with the same power through enchanting means of the normalization factor.

d) OFDM Technique: To have an efficient and effective frequency spectrum utilization is necessary. The Wi-MAX network system specification consist of OFDM block that provides frequency diversity and it consider to be base of the physical layer. Provides multiple transmission of signal at a time through using different sub-carriers.

Figure 1 represent the proposed MIMO-WiMAX architecture, where the model contains three major components such as; transmitter block shown by green color, channel shown by blue and receiver shown by orange color. Here, multiple transmitter and receiver antenna is considered for MIMO-WiMAX environment. The receiver and transmitter components contains source-encoder that followed by randomizer, modulator and demodulator, OFDM and, sub-components such as channel that modeled as Raleigh or Rician. 


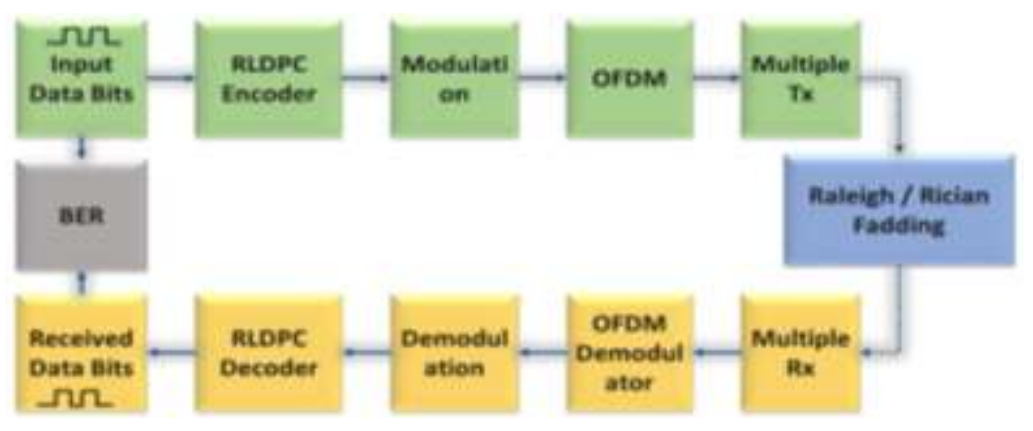

Figure 1. Proposed MIMO-WiMAX Architecture

\subsection{RLDPC CODES}

In this section, the author provides the considered principle and RLDPC encoding-decoding operation. The matrix of parity-check consist a least number of ones that lends to name, although these codes were neglected earlier phase due to the implementation complexity of hardware to run at real time. Therefore, researchers did not much focus of the LDPC codes, then recently LDPC codes has proven success at the data transmission. Here, the author consider robust code that has great transaction of flexibility because the code is able to support varity range of rates. Moreover, sparse matrix is used by the code which allows to store only elements of non-zero and locations instead of keeping all the matrix elements.

\section{- Encoding RLDPC}

The individual code in the RLDPC code-set is specified through a $A$ parity-check matrix of the size $p \times q$, where $A$ is given as;

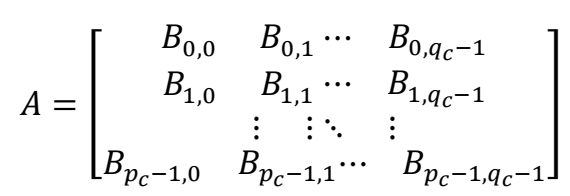

Where, $B_{i, j}$ denotes for a $s \times s$ zero matrix or a $s \times s$ permutation matrix with $s=\frac{q}{q_{c}}=\frac{p}{p_{c}}$ and $q_{c}$ is 24 . The process of permutations is done by circular right shifts.

Now, $A$ is further expanded through a intial value model matrix $A_{c p}$ and size of $p_{c} \times q_{c}$. Each of the positive entry $b(i, j)$ is replaced via a $B_{i, j}$ matrix with the equal size of circular shift to $b(i, j)$. Moreover, each of the negative or blank entry $b(i, j)$ is also replaced via a $B_{i, j}$ zero matrix.

Here, $A_{c p}$ defines standard for the highest length of code-word such as $q=2304$ at individual code-rate (CR). Also, $A_{c p}$ for various code-word $q^{\prime}$ length is acquired via scaling proportional shift size;

$$
b^{\prime}(i, j) \begin{cases}b(i, j) & b(i, j) \leq 0 \\ {\left[\frac{b(i, j), s_{f}}{s_{0}}\right]} & b(i, j)>0\end{cases}
$$

Where, $s_{0}=2304 / q_{c}$ and $s_{f}=\frac{q^{\prime}}{q_{c}}$

$A$ is divided so that it provide an efficient encoding;

$A=\left[\begin{array}{ccc}K_{(p-s) \times R q} & L_{(p-s) \times s} & T_{(p-s) \times(p-s)} \\ M_{s \times s} & N_{s \times s} & G_{s \times(p-s)}\end{array}\right]$

Here, the author define $H_{1}=G T^{-1} K+M, H_{2}=T^{-1} K$, and $H_{3}=T^{-1} L$.

So calculate $b$ using the $e$ info-word.

$$
\begin{aligned}
& b_{1}^{T}=H_{1} e \\
& b_{2}^{T}=H_{2} e+H_{3} b_{1}^{T}
\end{aligned}
$$


Consequently, the conforming computed code-word $u$ is given as;

$$
u=\left[e, b_{1}, b_{2}\right]
$$

The given values of code-word length $q$ and rate $R$ is considered in encoder implementation, which generates the $A$ matrix via the corresponding $A_{c p}$ intial matrix. Further calculate the matrices $H_{1}, H_{2}$, and $H_{3}$, and saves all these matrices. These matrices $H_{1}, H_{2}$, and $H_{3}$ are replenished at every simulation and further used in the encoding procedure.

- Decoding RLDPC

Decoding RLDPC is done by using belief propagation (BP) at the logarithmic domain. BP algorithms are normally expressed through the apprise message calculations on the factor-graph, which includes messages in among the variable nodes with respect to their neighboring nodes and vice versa [24, 25]. However, considering messages among the spans in a graph is only method for simplifying BP algorithm and it's based upon this neighboring factor-graph.

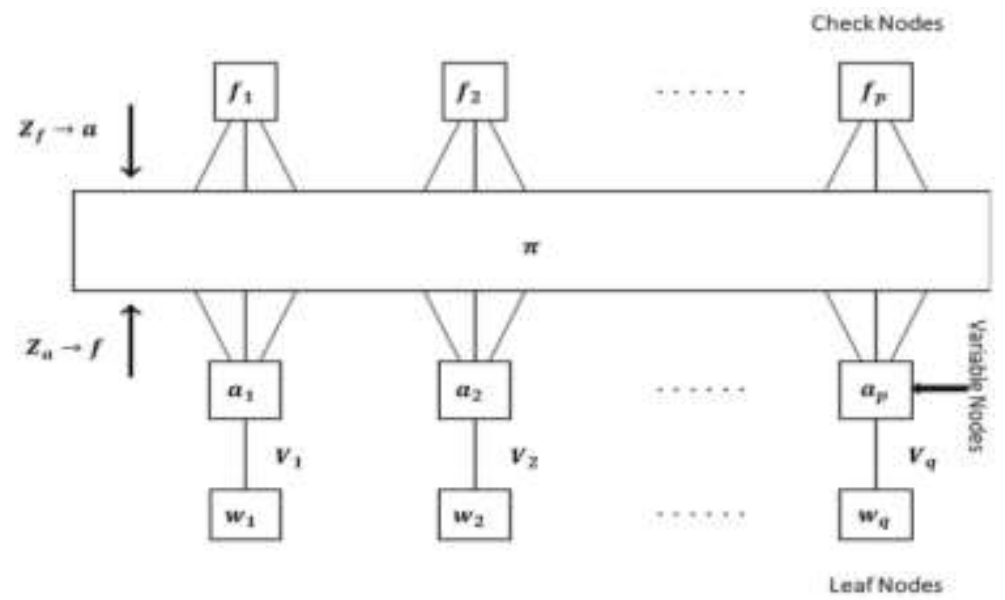

Figure 2. Belief Propagation based Factor Graph

Primarily the message $Z_{a} \rightarrow f$ are considered according to priority knowledge of channel $w$. Subsequently, schedule message is provided through check nodes and variable nodes with performing the process of ongoing marginalization.

$$
\widehat{V}_{I}=\left\{\begin{array}{c}
0, Z_{w_{I}} \rightarrow V_{I}+Z_{a_{I}} \rightarrow V_{I} \geq 0 \\
1, \text { otherwise }
\end{array}\right.
$$

Which is iterated to the maximum given iteration number or till a code-word is found. Leaf nodes are under hypothesis of same possible symbols of input and passing of message from leaf-nodes to the variable nodes are given by;

$$
w_{I}=-2 r_{I} / \mu_{\varepsilon}^{2}
$$

Where, $r_{I}$ denotes the $I$-th received symbol, noise variance is denoted by $\mu_{\varepsilon}^{2}$. The variable nodes from the update rule is given by;

$$
Z_{a} \rightarrow \beta=\sum_{i} Z_{\alpha_{i}} \rightarrow a
$$

So, updated rule for the check nodes is given via

$$
Z_{f} \rightarrow \beta=\tilde{\delta}\left(\sum_{i} \tilde{\delta}\left(\left|Z_{\alpha_{i}} \rightarrow f\right|\right)\right) \prod_{i} \operatorname{sign}\left(Z_{\alpha_{i}} \rightarrow f\right)
$$

Where; 


$$
\tilde{\delta}=-\log \left(\tanh \left(\frac{\alpha}{2}\right)\right)=\tilde{\delta}^{-1}
$$

The implementation of decoder is done via the function which provide $\hat{e}$ as estimated output of transmitted info-word $e$, whereas the $r$ denotes received vector and noise variance given by $\mu_{\varepsilon}^{2}$. The $w$ vector consist of the departing leaf-nodes message and transmitting upwards. The matrix $\mathcal{M}_{a f}$ consist of message variable and matrix $\mathcal{M}_{f a}$ consist of message check. The vector denoted by $\mathcal{M}_{a w}$ consist messages of the leaf nodes, which going towards down side. Initially, $\mathcal{M}_{a f}$ is initialize and $\varepsilon$ is computed till the max number of iteration $q$ or till code-word in not found. The update function of $\mathcal{M}_{f a}$ is given as;

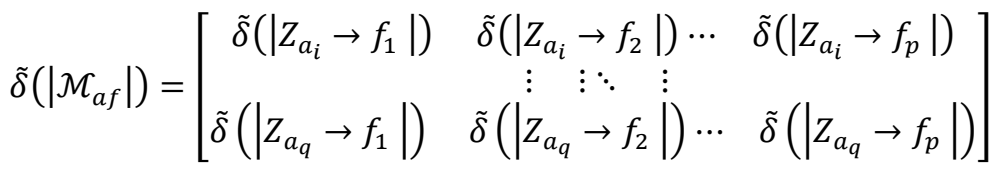

$$
\begin{aligned}
& \operatorname{Int}_{f}=\left(\left[\begin{array}{c}
\sum_{1}^{q} \tilde{\delta}\left(\left|Z_{a_{i}} \rightarrow f_{1}\right|\right) \\
\vdots \\
\sum_{1}^{q} \tilde{\delta}\left(\left|Z_{a_{i}} \rightarrow f_{p}\right|\right)
\end{array}\right][1 \ldots 1]\right) * A-\tilde{\delta}\left(\left|\mathcal{M}_{a f}\right|\right)^{T}
\end{aligned}
$$

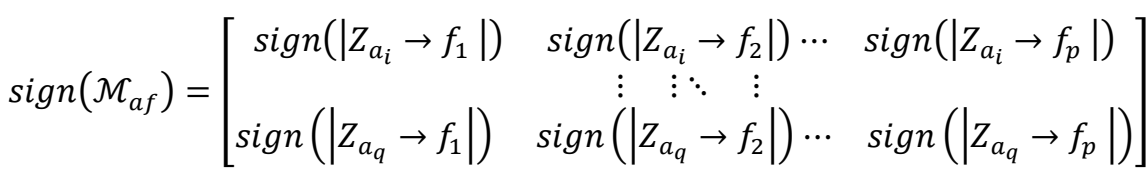

$$
\begin{aligned}
& \mathcal{M}_{f a}=\tilde{\delta}\left(\operatorname{Int} t_{f}\right) \cdot *\left(\left[\begin{array}{c}
\prod_{1}^{q} \operatorname{sign}\left(\left|Z_{a_{i}} \rightarrow f_{1}\right|\right) \\
\vdots \\
\prod_{1}^{q} \operatorname{sign}\left(\left|Z_{a_{i}} \rightarrow f_{p}\right|\right)
\end{array}\right][1 \ldots 1]\right) \cdot * \operatorname{sign}\left(\left|\mathcal{M}_{a f}\right|\right)^{T}
\end{aligned}
$$

Here, the update of matrices $\mathcal{M}_{a f}$ and $\mathcal{M}_{a w}$ is given by;

$$
\begin{aligned}
& \mathcal{M}_{a f}=\left(\left(\left[\begin{array}{c}
\sum_{1}^{p} Z_{f_{i}} \rightarrow a_{1} \\
\vdots \\
\sum_{1}^{p} Z_{f_{i}} \rightarrow a_{q}
\end{array}\right]+w\right)[1 \ldots 1]\right) * A^{T}-\mathcal{M}_{f a}{ }^{T} \\
& \mathcal{M}_{a w}=\left[\sum_{1}^{p} Z_{f_{i}} \rightarrow a_{1} \sum_{1}^{p} Z_{f_{i}} \rightarrow a_{2} \cdots \sum_{1}^{p} Z_{f_{i}} \rightarrow a_{q}\right]
\end{aligned}
$$

This can be marginalize if vector $\widehat{V}$ that is $\mathcal{M}_{a w}+w$ is less than zero. Moreover, the function $\tilde{\delta}$ is truncated in order to overcome the overflow and underflow errors;

$$
\tilde{\delta}(\alpha)=\left\{\begin{array}{cc}
12.5 & \alpha<10^{-5} \\
-\log (\tanh (0.5 \alpha)) & 10^{-5} \leq \alpha \leq 50 \\
0 & \alpha \geq 50
\end{array}\right.
$$

The process of iterative decoding is an efficient approach to minimize the problem with Bit interleaved coded - modulation (BICM) over an channels [26]. To decrease the excessive iterations of the decoding, here criteria of stopping has been introduced. BCIM is implemented via integrating the conform nodes at lower-side of RLDPC-factor-graph. Provides the robust act at different noisy channel condition and some modulation schemes. Therefore, the conform nodes update rule is given as;

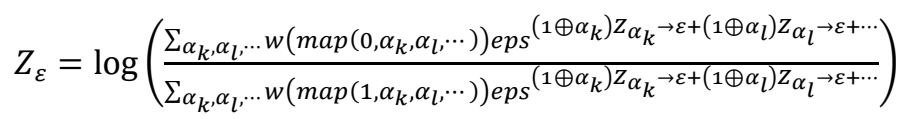

The modified decoder is used to estimate the output $\hat{e}$ of transmitted info-word $e$, whereas the $r$ is received vector with noise variance $\mu_{\varepsilon}^{2}$. The scheduling and initialization are modified in order to support the conform nodes. Besides, the simulation is carried out to validate code performance with respect to several test parameters that shows in the result analysis section. 


\section{RESULTS AND DISCUSSION}

In this section, the author provides result analysis on proposed MIMO based Wi-MAX system and it is analyzed in the platform of MATLAB/Simulink in terms of PAPR and BER. Information array is created by using the random number generator and the forward-error - correction have the RLDPC coding. However, the output of modulation is converted into the OFDM symbols, afterwards it transmitted over two antennas using the Rayleigh or Rician fading channel. While at the receiver side, the signal is received by two antennas and then demodulated through QAM and OFDM demodulator.

There are several standard LDPC code for different combination of code-rate is considered, possible code-rate such as; $1 / 2,3 / 4$, and 5/6. Also consider code-word lengths of 576 bits. Modulation using high order QAM provide added efficiency than the lower order QAM. However, the higher order modulation scheme requires the more signal power and increase the modulation complexity. Here, three modulation scheme is considered such as BPSK, QPSK and QAM-8 modulation. Moreover, the two scenario is considered where the scenario-A indicates for Rayleigh fading channel and scenario-B indicates for Rician fading channel.

\subsection{Scenario-A}

In this scenario-A, considered the Raleigh fading channel where Figure 3 shows PAPR reduction at different code-rates such as $1 / 2,3 / 4$, and 5/6. Here, the SNR of $5 \mathrm{~dB}$, modulation scheme BPSK and iteration 10 is considered, where it analyze that a 5/6 code-rate have less PAPR than the other code-rates. Figure 4 shows the BER analysis with different modulation scheme at $5 \mathrm{~dB}$ SNR, code-rate 5/6 and iteration 10 . The $\mathrm{y}$-axis shows the obtained BER and $\mathrm{x}$-axis shows the considered SNR value. Drastic reduction in BER is achieved at 7dB SNR using BPSK modulation, other than this, QPSK and QAM-8 has also shows reduction in BER with increasing number in SNR. Figure 5 shows the packet success rate (PSR) at different modulation scheme. The PSR analysis is done using BPSK, QPSK and, $Q A M-8$ modulation procedure, where the $\mathrm{y}$-axis shows obtained packet success ratio and $\mathrm{x}$-axis shows considered SNR value that ranges from -5 to $25 \mathrm{~dB}$.

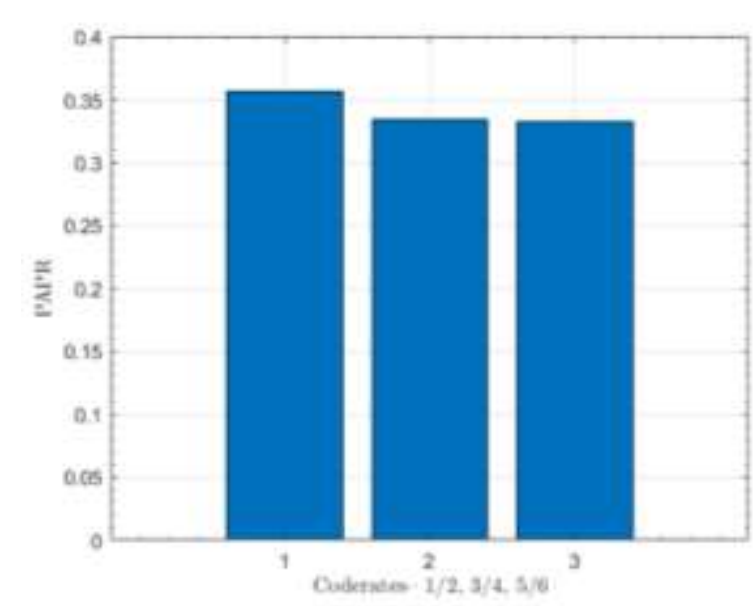

Figure 3. PAPR Reduction at different Code-rate

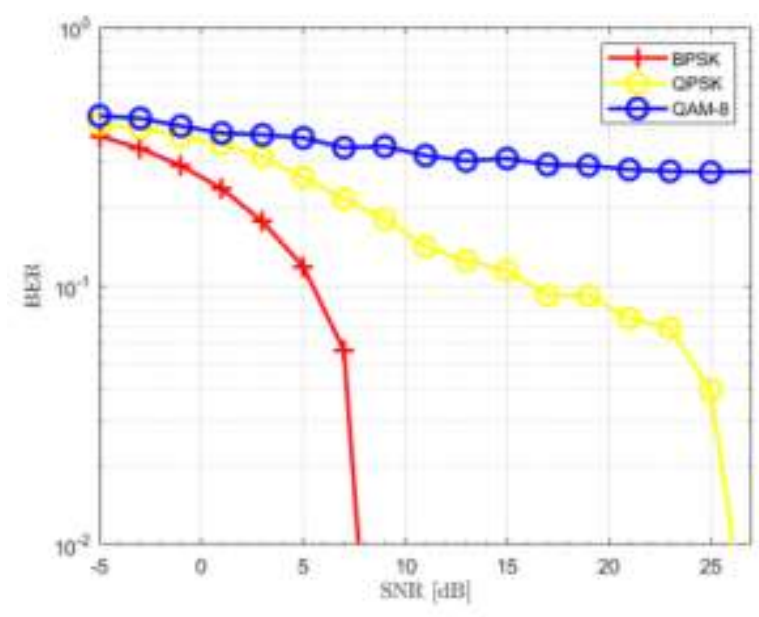

Figure 4. BER at different modulation scheme

BPSK modulation has achieved 0.89 average packet success ratio, whereas $Q P S K$ and $Q A M-8$ has achieved 0.79 and 0.65 average packet-success-ratio. The rate seems to be increasing as per increment in SNR value. In addition Table 1 shown, where presented BER analysis at different SNR and Iterations. Coderate 5/6 and modulation scheme BPSK is considered, as per increment in iteration the BER seems to decrease.

Table 1. BER analysis at different SNR and Iterations

\begin{tabular}{cccccc}
\hline SNR & $\mathbf{1}$ & $\mathbf{3}$ & $\mathbf{5}$ & $\mathbf{7}$ & $\mathbf{9}$ \\
\hline I-1 & 0.233333 & 0.177083 & 0.11875 & 0.052083 & 0.027083 \\
I-25 & 0.2375 & 0.177083 & 0.11875 & 0.05625 & 0.000167 \\
I-50 & 0.2375 & 0.177083 & 0.11875 & 0.05625 & 0 \\
\hline
\end{tabular}




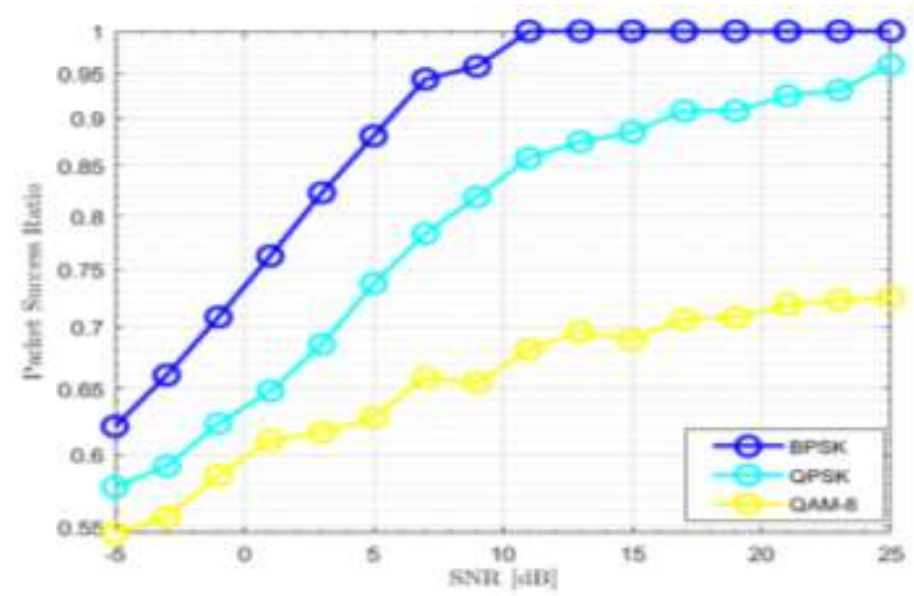

Figure 5. PSR at different modulation scheme

\subsection{Scenario-B}

This scenario-B show simulated result at Rician fading channel where Figure 6 shows the PAPR reduction at different code-rates, SNR is $5 \mathrm{~dB}$, iteration 10, code-length 576 and modulation scheme BPSK. Similarly as in scenario-A, here in Figure 7 shows the BER analysis with different modulation scheme. A very sudden reduction in BER using BPSK is seen in $9 \mathrm{~dB}$ SNR, whereas using QPSK it seen at $15 \mathrm{~dB}$ SNR, but however continuous reduction takes place with increasing SNR. Packet success rate (PSR) at different modulation scheme such as BPSK, QPSK and QAM-8 is shown in Figure 8, modulation scheme. The y-axis illustrates obtained packet success ratio and x-axis illustrates considered SNR value ranges from -5 to $25 \mathrm{~dB}$. The considered modulation scheme BPSK, QPSK and QAM-8 has achieved 0.91, 0.84 and 0.71 average packet success ratio. Table 2 shows BER analysis at different SNR and Iterations, where code-rate 5/6, codelength 576 and modulation scheme BPSK is considered. The BER comparison at different channel using BPSK modulation scheme is given in Figure 9, where the considered SNR value ranges from -5 to 9 dB. While at -3dB SNR the AWGN channel has got 7.97\% less BER compared to Rayleigh channel and Rayleigh channel has got $0.6 \%$ less BER compared to Rician channel.

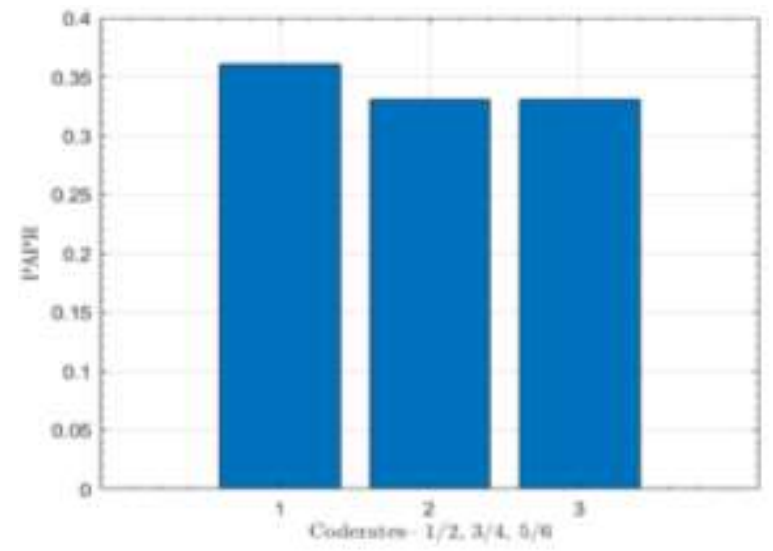

Figure 6. PAPR Reduction at different Code-rates

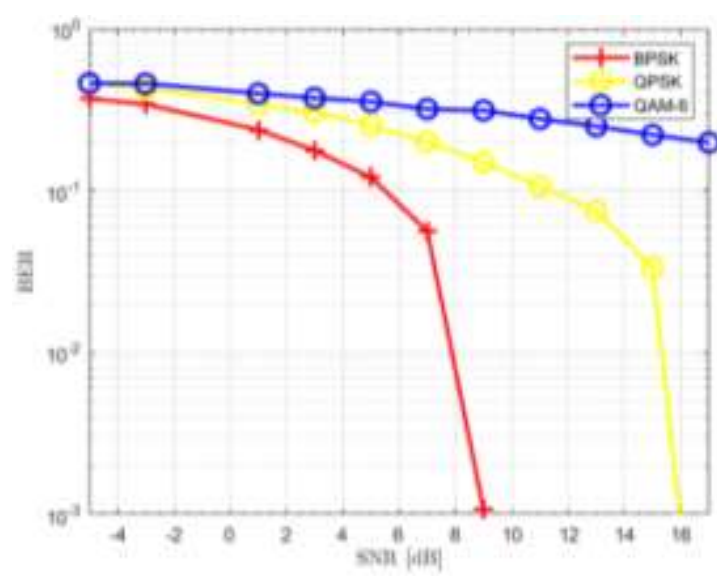

Figure 7. BER at different modulation scheme

Table 2. BER analysis at different SNR and Iterations

\begin{tabular}{cccccc}
\hline SNR & $\mathbf{1}$ & $\mathbf{3}$ & $\mathbf{5}$ & $\mathbf{7}$ & $\mathbf{9}$ \\
\hline $\mathbf{I - 1}$ & 0.2375 & 0.177083 & 0.11875 & 0.064583 & 0.029167 \\
$\mathbf{I - 2 5}$ & 0.2375 & 0.177083 & 0.120833 & 0.05625 & $6.25 \mathrm{E}-05$ \\
$\mathbf{I - 5 0}$ & 0.2375 & 0.177083 & 0.120833 & 0.05625 & 0 \\
\hline
\end{tabular}




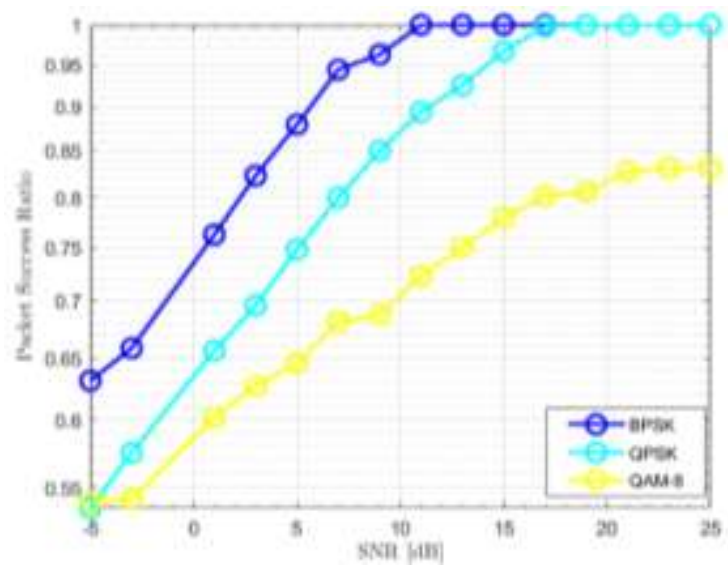

Figure 8. PSR at different modulation scheme

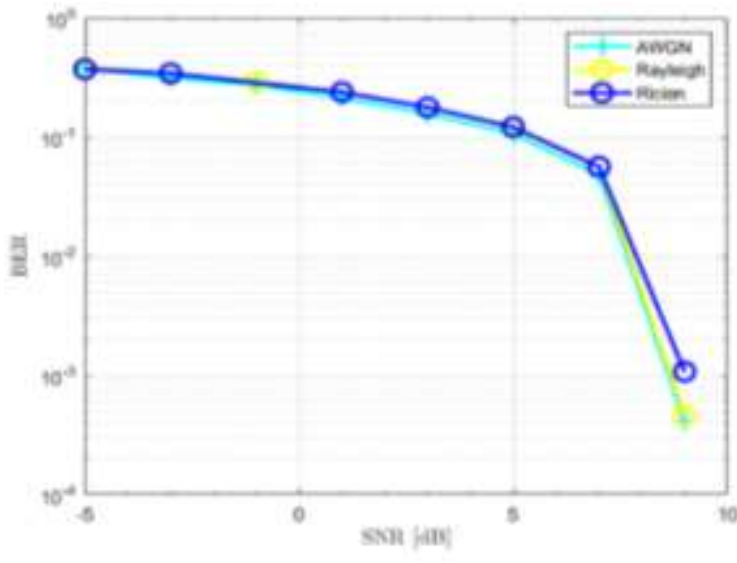

Figure 9. BER Comparison at different channel

Similarly, while considering $5 \mathrm{~dB}$ SNR the BER difference between AWGN channel and Rayleigh channel increases to $10.52 \%$. However, at 5dB SNR Rayleigh modulation has got $1.7 \%$ less BER compared to Rician channel, and there detailed representation of BER at different considered channel is provided in Table 3.

Table 3. BER at different considered channel

\begin{tabular}{cccccccc}
\hline SNR & -5 & -3 & 1 & 3 & 5 & 7 & 9 \\
\hline AWGN & 0.377083 & 0.3125 & 0.2125 & 0.15625 & 0.10625 & 0.047917 & 0.000405 \\
Rayleigh & 0.379167 & 0.339583 & 0.2375 & 0.177083 & 0.11875 & 0.05625 & 0.000463 \\
Rician & 0.36875 & 0.341667 & 0.2375 & 0.177083 & 0.120833 & 0.05625 & 0.001071 \\
\hline
\end{tabular}

\section{CONCLUSION}

The MIMO-OFDM system performance for IEEE 802.16e is analyzed at different modulations with the channel coding. In this study, the author majorly focusing on physical layer where minimization of the BER and PAPR is done by performing robust LDPC code. BP algorithms is used at decoding phase which is normally expressed through apprise message calculations on the factor graph, which includes communications amongst variable nodes with their neighboring nodes and vice versa. Result analysis section shows the effectiveness of our proposed model at both Rayleigh and Rician channel, where the results has shown at different code-rates, packet success rate, iterations and modulation schemes. We are able to minimize BER at every considered scenarios that shows the robustness of our proposed approach. In future work, MAC layer operation can be considered in order to provide optimized channel allocation at MIMO environment.

\section{REFERENCES}

[1] Part 16: Air interface for fixed and mobile broadband wireless access systems, IEEE Std 802.16Etm-2005, Feb 2006.

[2] A. Paulraj and D. Gore, Introduction to space-time wireless communications, Cambridge university press, 2003.

[3] S. A. Mujtaba, "MIMO signal processing: The next frontier for capacity enhancement," IEEE Custom Integrated Circuits Conference, 2003, pp 263-270.

[4] Z. Liu and G. B. Giannakis, "Space-time block coded multiple accesses through frequency-selective fading channels," IEEE Transactions on Communications, vol. 49, no. 6, pp. 1033-1044, Jun. 2001

[5] H. Sampath, S. TAlwar, V. Erceg and A. Paulraj, "A fourth-generation MIMO-OFDM broadband wireless system: design performance and field trial results," IEEE Communications Magazine, vol. 40, no. 9, pp 143-149, Sep. 2002.

[6] J. D. Kene and K. D. Kulat, "Performance evaluation of IEEE 802.16e WiMax physical layer," 2011 Nirma University International Conference on Engineering, Ahmedabad, Gujarat, 2011, pp. 1-4.

[7] C. Berrou, A. Glavieux, and P. Thitimajshima, "Near shannon limit error-correcting coding and decoding: Turbo codes," Proceeding of IEEE ICC, May 1993, pp. 1064-1070.

[8] Jagdish D. K. and Kishor D. K., "Performance evaluation of IEEE 802.16.e Wi-MAX physical layer" 2011 Nirma University International Conference on Engineering, Ahmedabad, India, Dec. 8-10, 2011, pp. 1-4.

[9] H.R Sadjapour and, F. Park, "Maximum A Posteriori Decoding Algorithms for the turbo codes," In digital wireless communication II, Proc of SPIE, vol. 4045, pp. 73-83, 2000. 
[10] Hao Wang, Hongwen Yang and Dacheng Yang, "Improved Log-MAP decoding algorithm for turbo-like codes," in IEEE Communications Letters, vol. 10, no. 3, pp. 186-188, Mar. 2006.

[11] E. Villebrum, P. Robertson, "A comparision of optimal and sub-optimal MAP decoding algorithms operating in the log-domain, " International Conference on Communications, Jun. 1995, pp.1009-1013.

[12] M. Taskaldiran, Richard C.S, "The modified max-log map turbo decoding algorithm by extrinsic information scaling for wireless application," In Wireless Technology, Applied DSP and VLSI Research Group, Department of Electronic Systems, University of Westminster, London, United Kingdom. pp 203-213, July 2009.

[13] J. D. Kene and K. D. Kulat, "WiMAX physical layer optimization by implementing SOVA Decoding algorithm," 2014 International Conference on Circuits, Systems, Communication and Information Technology Applications (CSCITA), Mumbai, 2014, pp. 179-183.

[14] Jagdish D. Kenea, Kishor D. Kulat, "Soft output decoding algorithm for turbo codes implementation in mobile Wimax environment," ICCCS-2012, 2012, pp. $666-673$.

[15] A. Dubey, P. Tavde and K. D. Kulat, "Study of OFDM system and performance investigation for various channel models," 2012 Third International Conference on Computing, Communication and Networking Technologies (ICCCNT'12), Coimbatore, 2012, pp. 1-8.

[16] S. R. Chaudhary, A. J. Patil and A. V. Yadao, "WLAN-IEEE 802.11ac: Simulation and performance evaluation with MIMO-OFDM," 2016 Conference on Advances in Signal Processing (CASP), Pune, 2016, pp. 440-445.

[17] Shubhangi R. Chaudhary, "Performance of WiMAX/ IEEE 802.16 with different modulation and coding," In International Conference on Advances in Information Technology and Mobile Communication, 2011, pp. 440-444.

[18] L. Alex and S. Amma, "Compact inverted u shaped slot triple band MIMO antenna for WLAN and WiMAX applications," 2018 Second International Conference on Inventive Communication and Computational Technologies (ICICCT), Coimbatore, 2018, pp. 1034-1036.

[19] M. Patidar, R. Dubey, N. Kumar Jain and S. Kulpariya, "Performance analysis of WiMAX 802.16e physical layer model," 2012 Ninth International Conference on WOCN, Indore, 2012, pp. 1-4.

[20] M. A. Mohamed, and H. M. Abd-El-Atty, "Performance simulation of IEEE 802.16e WiMAX physical layer," in 2nd IEEE International Conference on Information Management and Engineering, Chengdu, 2010, pp. 661-668.

[21] Y. Aimer, B. S. Bouazza, "PAPR reduction performance in WIMAX OFDM systems using interleavers with downward-compatibility," 2018 IEEE International Symposium on Circuits and Systems (ISCAS), 2018, pp. 1-5.

[22] I. Ahmad and D. Habibi, "A novel mobile WiMAX solution for higher throughput," 2008 16th IEEE International Conference on Networks, New Delhi, 2008, pp. 1-5.

[23] V. Dhilip Kumar, Phidahunlang Chyne "Performance analysis of hybrid WiMAX/DSRC scenarios for vehicular communication environment," Microsystem Technologies, vol. 23, no. 9, pp 4231-4236, Sep. 2017

[24] Hua Li, Hong Ding, Linhua Zheng, Ziwei Lei, Hui Xiong and Tan Wang, "An efficient scheduling scheme for layered belief propagation decoding of regular LDPC codes," Ultra-Modern Telecommunications and Control Systems and Workshops (ICUMT), Lisbon, 2016, pp. 397-400.

[25] T. Hehn, J. B. Huber and S. Laendner, "Improved iterative decoding of LDPC codes from the IEEE WiMAX standard," 2010 International ITG Conference on Source and Channel Coding (SCC), Siegen, 2010, pp. 1-6.

[26] S. Zhang, J. Li and J. Chen, "Three Simple Iterative Decoding Schemes for BICM-ID," 2010 International Conference on Biomedical Engineering and Computer Science, Wuhan, 2010, pp. 1-4. 